\title{
On Selfish and Altruistic Coalition Formation in Cognitive Radio Networks
}

\author{
Zaheer Khan, Janne Lehtomäki and Matti Latva-aho \\ Center for Wireless Communications (CWC), \\ University of Oulu, Finland.
}

\author{
Luiz A. DaSilva \\ Centre for Telecommunications Value-chain Research, \\ Trinity College Dublin, Ireland.
}

\begin{abstract}
We formulate the sensing-throughput tradeoff problem for distributed cognitive radio (CR) networks as a coalition formation game. Formation of coalitions enables the CRs to increase their achievable throughput, under the detection probability constraint, while also taking into account the overhead in sensing reports combining. In the proposed game, CRs form coalitions either to increase their individual gains (selfish coalition formation) or to maximize the overall gains of the group (altruistic coalition formation). We find that the altruistic coalition formation solution yields significant gains in terms of reduced average false alarm probability and increased average throughput per CR as compared to the selfish and non-cooperative solutions. Given a target detection probability for a coalition, we also propose an SNR dependent target detection probability for individual CRs in a coalition and analyze its impact on the average throughput per CR. Finally, we also analyze the impact of the cost of distributed cooperative sensing on the cooperative strategies of CRs.
\end{abstract}

\section{INTRODUCTION}

Cognitive radio (CR) technology has been recently proposed to improve the efficient utilization of radio spectrum. A CR utilizes spectrum opportunistically by monitoring the environment to reliably detect the primary (licensed) user signals and operating whenever the primary user (PU) is absent. The detection of PU signals is called spectrum sensing. As with any detection problem, the two types of errors associated with spectrum sensing are false alarms and missed detections. The lower the missed detection probability, the better the PU is protected. However, to increase the achievable throughput of the CRs, the false alarm probability must also be low. Thus, there exists an inherent tradeoff between sensing capability and achievable throughput for a cognitive radio network [1].

Cooperative sensing has been shown to increase the reliability of spectrum sensing [2]. In cooperative spectrum sensing, each CR performs spectrum sensing and sends its sensing report to a data collector known as the fusion center. To reduce signaling cost, the report may be binary (hard decision), consisting of zeros (PU not present) and ones (PU present). Hard decisions may be combined at the fusion center using, for example, "OR", "AND" and "MAJORITY" rules [1]. However, cooperative sensing among distributed CRs requires individual CRs to interact with each other without a centralized fusion center.

In this paper, using a coalition game-theoretic framework we devise distributed cooperative strategies for CRs that are either selfish or altruistic. We choose a coalition formation framework as it provides tools for the radios to decide which coalitions to form to achieve their goals more efficiently via cooperation [3]. A coalition is a set of distinct, autonomous agents or players which may cooperate in order to increase their individual gains, which we denote as selfish cooperation. Or they may cooperate to maximize the overall gains of the group, which we call altruistic cooperation [4]. A key question this paper tries to address is how to coordinate distributed cognitive radios to perform cooperative sensing to

This work was funded by the Finnish Funding Agency for Technology and Innovation (TEKES). minimize their false alarm probabilities and therefore increase their achievable throughput, under a probability of detection constraint, while also taking into account the overhead in combining sensing reports.

Two different approaches in analyzing distributed cooperative spectrum sensing are presented in [5] and [6]. In [6], an evolutionary game theoretic framework is used to analyze the interactions among distributed selfish CRs in cooperative sensing. It is assumed in [6] that the selfish CRs overhear the detection results from the other CRs and can free ride by refusing to take part in spectrum sensing. Hence [6] models the spectrum sensing problem as a non-cooperative game. Distributed coalition formation for cooperative spectrum sensing CRs is the topic of [5]. Using a merge-and-split based coalition formation game model, the authors in [5] analyze the average missed detection probability per CR.

In this paper, we utilize coalition game theory to model the sensing-throughput tradeoff problem in distributed cognitive radio networks. Unlike [5], we propose a value function that encourages collaborating CRs to minimize their false alarm probabilities for a given target PU detection probability $\left(\tilde{P}_{d}\right)$. This is an important requirement for coexistence with the PU, otherwise CRs will not be allowed to operate in the PU band [7]. Moreover, in contrast with [5], the coalition formation game proposed in this paper also takes into account the overhead in combining sensing reports within a coalition. Formation of coalitions enables the CRs to reach throughputefficient and self-organizing stable spectrum sensing network partitions. Using simulations we assess the performance of the proposed altruistic and selfish coalition formation solutions in terms of gains in increased average throughput per CR as compared to a non-cooperative strategy. We also determine average maximum coalition sizes for both altruistic and selfish coalition formation solutions.

The rest of the paper is organized as follows. Section II presents the system setup, while Section III introduces the proposed coalition formation game for distributed spectrum sensing. Section IV presents simulation results and an analysis of the proposed coalition formation game, and Section $\mathrm{V}$ concludes the paper.

\section{System Setup}

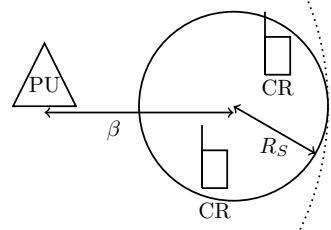

Fig. 1. Topology of a cognitive radio network.

The system setup used in this paper includes a PU transmitter and a distributed CR network of $n$ active CRs (transmit- 
ter/receiver pairs). The CRs are uniformly and independently distributed in a circle with radius $R_{s}$ and centered at the coordinates $(\beta, 0)$. The PU (primary user transmitter) is at coordinates $(0,0)$ as shown in Fig. 1. This corresponds to, for example, using only downlink frequencies for CR access. The PU and CRs are both assumed to use a time slotted system with perfect time-synchronization, and one transmission by PU corresponds to one time slot [8], [9]. ${ }^{1}$ The CRs use the beginning of each slot for sensing. We assume that $n$ cognitive radios employ energy detection to make PU detection observations in the frequency band they are monitoring. In order to detect the PU, each CR can either sense the spectrum on its own (non-cooperative strategy) or it can perform cooperative sensing by forming coalitions with the other CRs (cooperative strategy).

Let us represent the signal power received by $\mathrm{CR} i$ by:

$$
P_{i}=\frac{P_{P U} \kappa}{d_{i}^{\alpha}},
$$

where $P_{P U}$ is the PU's signal power, $d_{i}$ is the distance between the PU and the $i$ th $\mathrm{CR}, \alpha$ is the path-loss exponent and $\kappa$ is a scalar. The received PU's signal-to-noise ratio (SNR) at the $\mathrm{CR} i$ is given by:

$$
\gamma_{i}=10 \log \frac{P_{i}}{\sigma^{2}},
$$

where $\sigma^{2}$ represents noise power. In our system setup we assume complex-valued PSK PU signal and circular symmetric complex Gaussian (CSCG) noise. For the CSCG noise case the probability of false alarm of CR $i$ for a chosen detection threshold $\lambda_{i}$ is given by [1], [10]:

$$
P_{f, i}\left(\lambda_{i}\right)=Q\left(\left(\frac{\lambda_{i}}{\sigma^{2}}-1\right) \sqrt{N}\right)
$$

where $\mathrm{Q}($.$) is the tail probability for the standard normal$ distribution and $N$ represent the time-bandwidth product and is given as $N=\tau_{s} W$, where $\tau_{s}$ is the sensing duration and $W$ is the measurement bandwidth. For a chosen threshold $\lambda_{i}$, the probability of detection of CR $i$ is approximated by [1], [10]:

$$
P_{d, i}\left(\lambda_{i}, \gamma_{i}\right)=Q\left(\left(\frac{\lambda_{i}}{\sigma^{2}}-\gamma_{i}-1\right) \sqrt{\frac{N}{2 \gamma_{i}+1}}\right) .
$$

To protect the PU against harmful interference from the CRs, the detection probability is fixed at a desired target value, $\tilde{P}_{d}$. In practise, $\tilde{P}_{d}$ is required to be close to $1[11]$. The probability of false alarm of each CR $i$ for the targeted $\tilde{P}_{d}$ is rewritten as:

$$
P_{f, i}\left(\tilde{P}_{d}, \gamma_{i}\right)=Q\left(\sqrt{2 \gamma_{i}+1} Q^{-1}\left(\tilde{P}_{d}\right)+\sqrt{N} \gamma_{i}\right) .
$$

It may be seen from (5) that a high detection probability requirement may lead to a high false alarm probability for an individual CR if its $\gamma_{i}$ is low, thus reducing the achievable throughput of that CR. In this case, the individual CRs may interact to form coalitions to help decrease the false alarm probability. Within a coalition, sensing decisions of individual CRs are transmitted over the narrowband common control channel to a CR selected as a coalition head. An OR fusion rule is used by the coalition head to combine the individual CR sensing decisions within a coalition. ${ }^{2}$ The OR rule is a simple decision rule explained as follows: if one or more out of $|\mathbf{S}|$ CRs in a coalition detects the PU, the final decision for the coalition declares a PU is present, where $|\mathbf{S}|$ represents the number of CRs in a coalition $\mathbf{S}$. CRs in any coalition $\mathbf{S}$ decide to transmit or not based on the final combined sensing decision of the coalition head. Therefore, the probabilities of detection and false alarm of a coalition head are also the probabilities of detection and false alarm of each CR $i$ that is a member of $\mathbf{S}$. Assuming that all decisions are conditionally independent,

\footnotetext{
${ }^{1}$ In this approach, the PU is either present in the whole time slot, or absent in the whole time slot.

${ }^{2}$ Although the optimal decision fusion rule is the Chair-Varshney rule [12] for simplicity of implementation we analyze the OR rule.
}

3 then using the OR rule, the detection probability of the coalition $\mathbf{S}$ is given as:

$$
P_{d, \mathbf{S}}=1-\prod_{i=1}^{|\mathbf{S}|}\left(1-P_{d, i}\right) .
$$

For a given $\tilde{P}_{d}$, the individual CR's target probability of detection in a coalition using the OR fusion rule is written as (assuming same target probability of detection for every CR [6], [10]):

$$
\tilde{P}_{d, i}=1-\left(1-\tilde{P}_{d}\right)^{\frac{1}{|S|}} \text {. }
$$

However, it may happen that some of the CRs have better received PU SNR than the others. To gain from this SNR diversity, we propose the weighted target probability of detection for any CR $i \in \mathbf{S}$. The weighted target probability of detection modifies $\tilde{P}_{d, i}$ in (7) and takes a sensing CR's received PU SNR $\gamma_{i}$ into consideration. Weighted target probability of detection is given as:

$$
\tilde{P}_{d, i}^{w}=1-\left(1-\tilde{P}_{d}\right)^{\frac{\gamma_{i}}{\sum_{j \in \mathbf{S}} \gamma_{j}}} .
$$

The probability of false alarm of each CR $i$ for the $\tilde{P}_{d, i}^{w}$ is written as:

$$
P_{f, i}\left(\tilde{P}_{d, i}^{w}, \gamma_{i}\right)=Q\left(\sqrt{2 \gamma_{i}+1} Q^{-1}\left(\tilde{P}_{d, i}^{w}\right)+\sqrt{N} \gamma_{i}\right)
$$

Using the OR rule, the false alarm probability $P_{f, \mathbf{S}}$ of the coalition $\mathbf{S}$ is given as:

$$
P_{f, \mathbf{S}}=1-\prod_{i=1}^{|\mathbf{S}|}\left(1-P_{f, i}\right) .
$$

It may be seen from (9) that the CRs with low values of $\gamma_{i}$ have incentives to form coalitions as it helps to decrease $P_{f, i}$ due to the $Q^{-1}\left(\tilde{P}_{d, i}^{w}\right)$ term. However, the coalitional false alarm probability given by (10) is an increasing function of coalition size $|\mathbf{S}|$.

\section{CoAlition GAme-Theoretic Framework For Distributed COOPERATIVE SENSING}

\section{A. Preliminaries}

Let $\mathbf{N}$ denote the set of players (CRs) playing the coalition game, $\mathbf{N}=\{1,2, \ldots, n\}$. A coalition, $\mathbf{S}$, is a subset of $\mathbf{N}, \mathbf{S} \subseteq$ $\mathbf{N}$. An individual non-cooperating player is called a singleton coalition and the set $\mathbf{N}$ is called the grand coalition, where all players cooperate. The utility of a coalition in a coalition game is called the coalition value and is denoted by $v$. Coalitions are assumed to be non-overlapping, i.e., CRs are members of at most one coalition.

The most common form of a coalition game is the characteristic function form. In the characteristic function form (CFF) of coalition games, utilities achieved by the players in a coalition are unaffected by those outside it.

Definition 1: A non-transferable utility (NTU) game is a coalition game in CFF, in which the value $v(\mathbf{S})$ of a coalition $\mathbf{S}$ cannot be arbitrarily divided among the coalition's players. In such games, each player will have its own value within a coalition $\mathbf{S}$. The value function $\varphi_{i}(\mathbf{S})$ represents the value of player $i$ that belongs to a coalition $\mathbf{S}$.

\section{B. Throughput of a CR}

For a CR network with periodic spectrum sensing, each slot consists of sensing duration $\tau_{s}$ and data transmission duration $T_{f}-\tau_{s}$ [1]. To focus on the coalition formation game for the sensing-throughput tradeoff problem in distributed CR networks we assume that the entire PU band is divided into $K$ sub-bands and, when the PU is absent, each CR operates

\footnotetext{
${ }^{3}$ This means that the measurements of CRs are independent, but that for each CR the same hypothesis $\left\{H_{0}, H_{1}\right\}, H_{0}=\mathrm{PU}$ not present and $H_{1}=\mathrm{PU}$ present, applies.
} 
exclusively in one of the $K$ sub-bands. ${ }^{4}$ This assumption is in line with other works in the literature [6], [13]. For the non-cooperative case, the average throughput of the CR $i$ is approximated by [1], [6]:

$$
R_{i}=P_{H_{0}}\left(1-\frac{\tau_{s}}{T_{f}}\right)\left(1-P_{f, i}\right) r_{i},
$$

where $P_{H_{0}}$ is the probability of PU absent, $T_{f}$ represents the total slot length and $r_{i}$ represents the transmission rate of the $\mathrm{CR} i$ to its receiver when the PU is absent. However, when the CRs decide to form a coalition then the entire coalition cannot transmit data until sensing reports are collected and the final combined sensing decision is transmitted to all the coalition members. One simple method of sensing reports collection by the coalition head can be stated as follows: The coalition head grants a contention free channel to individual cognitive radios by polling them (using their identity numbers) for transmitting their local decisions. The coalition head may employ a round-robin scheduler [11], [14], and on being polled, a CR transmits its local decision to the coalition head. In this sensing reports collection there is cost in terms of time delay in data transmissions of a coalition due to the overhead in combining sensing reports. This cost generally increases with the number of coalition forming CRs, as more decisions need to be reported to the coalition head [11], [14]. The average throughput of the CR $i$ considering the cost in terms of overhead in combining sensing reports within a coalition is approximated as:

$$
\hat{R}_{i}=P_{H_{0}}\left(1-\frac{\tau_{s}}{T_{f}}-\frac{\tau_{c}}{T_{f}}(|\mathbf{S}|-1)\right)\left(1-P_{f, \mathbf{S}}\right) r_{i},
$$

where $\tau_{c}$ is the time spent on reporting a sensing decision to the coalition head. For a target detection probability, $\tilde{P}_{d}$, the CRs may form coalitions to gain in terms of reduced false alarm probability and therefore increase their average throughput given by (12). The coalition forming CRs may also increase their average throughput by reducing their sensing time $\tau_{s}$ via joint coalitional sensing. However, we note that for the distributed spectrum sensing problem when the coalition forming CRs are allowed to vary sensing time $\tau_{s}$, it generates significant uncertainties in the coalition values. For instance, if two or more CRs reduce their sensing time $\tau_{s}$ (or in other words increase their data transmission time) via forming the coalition $\mathbf{S}$ then it may happen that the CRs in $\mathbf{S}$ start transmitting data while some CRs outside $\mathbf{S}$ may still be sensing. This may lead to a change in the coalition value (in terms of false alarm probabilities) of the CRs outside the coalition $\mathbf{S}$. To avoid this uncertainty in the coalition value due to $\mathrm{CR}$ transmissions we fix the sensing duration $\tau_{s}$ for each individual $\mathrm{CR}$.

\section{Coalition Value with Non-transferable Utility}

For a target detection probability, $\tilde{P}_{d}$, and the fixed sensing duration $\tau_{s}$, the coalition value $v(\mathbf{S})$ must characterize the incentives to form coalitions in terms of the decreased false alarm probability $P_{f}$ s of the coalition. Moreover, the coalition value must also take into account the cost in terms of delay in data transmissions of a coalition due to the overhead in sensing reports combining. A suitable coalition value that satisfies the above requirements is given by:

$$
\begin{array}{r}
v(\mathbf{S})=\left(\varphi_{1}(\mathbf{S}), \varphi_{2}(\mathbf{S}), \ldots, \varphi_{|\mathbf{S}|}(\mathbf{S})\right), \\
=\left(\hat{R}_{1}, \hat{R}_{2}, \ldots, \hat{R}_{|\mathbf{S}|}\right),
\end{array}
$$

where $\varphi_{i}(\mathbf{S})$ denotes the average throughput for CR $i$ given by (12), for $i=1,2, \ldots,|\mathbf{S}|$. In the proposed coalition formation game, each $\mathrm{CR}$ has its own value within a coalition and its non-transferable due to the following reasons. 1) Indivisible false alarm probability: The probability of false alarm $P_{f, i}$ of each CR $i$, where $i \in \mathbf{S}$, is also given by the probability of

\footnotetext{
${ }^{4}$ We do not analyze scheduling policies in this work and assume a simple and predetermined orthogonal sub-band allocation policy for the CRs when the primary user band is free for access
}

false alarm of the coalition $\mathbf{S}$, i.e., $P_{f, \mathbf{S}}$ (as explained in Section II) and it cannot be divided among the radios. 2) Indivisible cost: Each CR incurs the same cost in terms of overhead in combining sensing reports, i.e., $\frac{\tau_{c}}{T_{f}}(|\mathbf{S}|-1)$, and this cost cannot be divided among the CRs. 3) Indivisible bandwidth: Finally, each CR operates exclusively in one of the $K$ subbands. Therefore, they cannot arbitrarily divide the spectrum among themselves.

Therefore, each $\mathrm{CR}$ will have its own value within the coalition $\mathbf{S}$ and hence, the proposed game is an NTU game.

The decisions to form coalitions by the CRs are based on consensus, i.e., a coalition is formed only if it is acceptable to everyone involved. We also assume that CRs are myopic, i.e., CRs care only about their current payoffs.

\section{Selfish Coalition Formation}

Definition 2: Internal stability means that no $\mathrm{CR}$ has an incentive to leave its coalition to become a singleton (individual non-cooperative CR), i.e., $\boldsymbol{\varphi}_{i}\left(\mathbf{S}_{\mathbf{1}}\right) \geq v(\{i\}), \forall i \in \mathbf{S}_{\mathbf{1}}$.

We assume that CRs are individually rational, i.e., CRs seek to maximize their payoffs, conditional on feasibility. Therefore, for the selfish coalition formation, the merge of two coalitions only occurs when all CRs in the new coalition $\mathbf{S}$ are at least as well off through the merge as they were before it. Mathematically speaking, coalitions $\mathbf{S}_{1}$ and $\mathbf{S}_{2}$ will merge to form $\mathbf{S}$ only: if $\forall i, j$, where $i \in \mathbf{S}_{1}$ and $j \in \mathbf{S}_{2}$, $\left(\varphi_{i}(\mathbf{S})-\varphi_{i}\left(\mathbf{S}_{1}\right)\right) \geq 0$ and also $\left(\varphi_{j}(\mathbf{S})-\varphi_{j}\left(\mathbf{S}_{2}\right)\right) \geq 0$. Due to this coalition formation condition, whenever CRs agree to form $\mathbf{S}$ the new coalition is internally stable, i.e., no CR has an incentive to become a singleton (an individual non-cooperative CR).

\section{E. Altruistic Coalition Formation}

The model of selfish coalition formation discussed above is in line with much of the coalition formation literature, which assumes that users form coalitions to maximize their individual payoffs. However, it is equally interesting to investigate the question whether and how the achievable throughput of CRs will change if CRs are assumed to be "not entirely selfish". Intuitively, we want to model that when two or more CRs propose to form a new coalition $\mathbf{S}$ they do take into account one another's welfare. Before studying the altruistic coalition formation, we first define the concept of altruistic contribution of a coalition as [4]:

Definition 3: Let $\mathbf{S}_{1}$ and $\mathbf{S}_{2}$ be two disjoint coalitions. The altruistic contribution of $\mathbf{S}_{1}$ to $\mathbf{S}$, where $\mathbf{S}=\mathbf{S}_{1} \cup \mathbf{S}_{2}$, is $a_{\mathbf{S}_{1}}(\mathbf{S})=\sum_{i \in \mathbf{S}_{2}}\left(\varphi_{i}(\mathbf{S})-\varphi_{i}\left(\mathbf{S}_{2}\right)\right)$; the altruistic contribution of $\mathbf{S}_{2}$ to $\mathbf{S}$ is $a_{\mathbf{S}_{2}}(\mathbf{S})=\sum_{i \in \mathbf{S}_{1}}\left(\left(\varphi_{i}(\mathbf{S})-\varphi_{i}\left(\mathbf{S}_{1}\right)\right)\right.$; and the sum of altruistic contributions of $\mathbf{S}_{1}$ and $\mathbf{S}_{2}$ is $\hat{a}(\mathbf{S})=a_{\mathbf{S}_{1}}(\mathbf{S})+a_{\mathbf{S}_{2}}(\mathbf{S})$.

In simple words, the altruistic contribution of coalition $\mathbf{S}_{1}$ to $\mathbf{S}$ represents the change in the value of the CRs in $\mathbf{S}_{2}$, when the CRs in $\mathbf{S}_{1}$ are added to the coalition $\mathbf{S}$. It can be easily seen that if any proposed coalition $\mathbf{S}$ has $\hat{a}(\mathbf{S})>0$, then the merger of $\mathbf{S}_{1}$ and $\mathbf{S}_{2}$ to form $\mathbf{S}$ would do more good than harm to the overall value of the coalition $\mathbf{S}$. Formally, we assume that to maximize the achievable throughput of the proposed coalition $\mathbf{S}$, an altruistic coalition decides to form the coalition $\mathbf{S}$ whenever

$$
\hat{a}(\mathbf{S})>0 .
$$

\section{F. Coalition Formation Game Model}

To model all $n$ CR coalitions, we define coalition structures as follows:

Definition 4: A coalition structure is a partition of $\mathbf{N}$ into exhaustive and disjoint subsets, where each subset is a coalition. The set of all possible coalition structures is denoted as $\mathbf{C}$, where $\mathbf{C}=\left\{\mathbf{C}_{1}, \mathbf{C}_{2}, \ldots, \mathbf{C}_{|\mathbf{C}|}\right\}$.

In this subsection, we introduce a coalition formation model for distributed cooperative spectrum sensing in CR networks where multiple distributed CRs coexist and opportunistically access the spectrum. To incorporate slow changes in the network configuration (for e.g. due to CR mobility), the first round of coalition formation game restarts after some time $T$ 
after reaching equilibrium. This time $T$ may be assigned according to variations in the network configuration. We assume that during one round of coalition formation the received PU's SNR, i.e., $\gamma_{i}$, and the transmission rate $r_{i}$ of each CR $i$ does not change. At the very beginning of each round of the coalition formation game, the distributed CR network is composed of all singleton coalitions, i.e., non-cooperative CRs. The coalition formation game involves five steps. The five steps of the proposed model are summarized as follows:

1) Node discovery: Discover the CRs within the network.

2) Initialization: Each individual $\mathrm{CR}$ computes its received PU's SNR, i.e., $\gamma_{i}$.

3) Coalition formation proposal: a) Each coalition, with probability $p$, proposes a new coalition structure [15]. In this process, one of the CRs in the coalition acts on behalf of the entire coalition. (In the case of singleton coalitions, each singleton coalition, i.e., each CR, individually proposes a new coalition structure with some probability $p$; when two or more CRs form a coalition $\mathbf{S}$, then any CR within $\mathbf{S}$ is selected as a coalition head to propose a new coalition structure with some probability $p$ on behalf of that coalition and also to combine the individual $\mathrm{CR}$ sensing decisions within the coalition.) b) The evolution from one coalition structure to the next can only occur through the merging of two existing coalitions. For instance, any coalition head of any existing coalition $\mathbf{S}_{\mathbf{1}}$ may randomly select another coalition $\mathbf{S}_{2}$ and propose to merge with it, forming $\mathbf{S}_{\mathbf{1}} \cup \mathbf{S}_{\mathbf{2}}=\mathbf{S}$.

4) Coalition formation decision: a) When the CRs are assumed to be selfish then they form a coalition if $\forall i, j$, where $i \in \mathbf{S}_{1}$ and $j \in \mathbf{S}_{2}, \quad\left(\varphi_{i}(\mathbf{S})-\varphi_{i}\left(\mathbf{S}_{1}\right)\right) \geq 0$ and also $\left(\varphi_{j}(\mathbf{S})-\varphi_{j}\left(\mathbf{S}_{2}\right)\right) \geq 0$.

b) When the CRs are assumed to be altruistic then they form a coalition if $\hat{a}(\mathbf{S})>0$. (see Section III-D and III-E for the details).

The steps 3 and 4 of the coalition formation are repeated until all the coalitions have made their coalition formation decisions, resulting in a final stable coalition structure $\mathbf{C}_{F}$.

5) Coalitional spectrum sensing: Each CR with in a coalition computes its local sensing decision and transmits it to the coalition head over the common control channel. Coalition head combine the local sensing decisions (including its own sensing decision) using the OR decision fusion rule.

The negotiation process described above can be achieved using a common control channel where CRs can exchange coalition formation messages to perform the proposed distributed coalition formation.

\section{Game Analysis and Simulation Results}

\section{A. Stable Coalition Structures}

As CRs form self-organizing spectrum sensing coalition structures. It is interesting to analyze, under what conditions the coalition formation process will reach a stable coalition structure (where no two coalitions have an incentive to merge anymore). A stable coalition structure will evolve if the coalition formation process reaches an equilibrium state. In an equilibrium state no two coalitions have an incentive to merge anymore.

1) Selfish Coalition Formation: For the proposed selfish coalition formation, a coalition structure state $\mathbf{C}^{*}$ is an equilibrium state if it satisfies the following condition:

$\forall \mathbf{S}_{j}, \mathbf{S}_{k, k \neq j} \in \mathbf{C}^{*}$, for some $i \in \mathbf{S}_{j}\left(\varphi_{i}\left(\mathbf{S}_{j} \cup \mathbf{S}_{k}\right)-\varphi_{i}\left(\mathbf{S}_{j}\right)\right)<0$, or for some $i \in \mathbf{S}_{k}\left(\varphi_{i}\left(\mathbf{S}_{k} \cup \mathbf{S}_{j}\right)-\varphi_{i}\left(\mathbf{S}_{k}\right)\right)<0$.

The above stated condition ensures that no two coalitions in the prevailing coalition structure $\mathbf{C}^{*}$ have an incentive to merge anymore.

The following simple fact proves that the selfish coalition formation process converges to an equilibrium state: In the proposed selfish coalition formation, if a certain coalition structure is not an equilibrium state, there must exist at least two coalitions that can decide to merge to improve their value functions. As long as such two coalitions exist, the coalition structure changes to another coalition structure or else this procedure stops at an equilibrium state.

2) Altruistic Coalition Formation: For the proposed altruistic coalition formation, a coalition structure $\mathbf{C}^{*}$ is an equilibrium state if it satisfies the following condition:

\section{$\forall \mathbf{S}_{j}, \mathbf{S}_{k, k \neq j} \in \mathbf{C}^{*}$, if $\hat{a}(\mathbf{S})>0$.}

The following simple fact proves that the altruistic coalition formation process converges to an equilibrium state: In the proposed altruistic coalition formation model, if a certain coalition structure state is not an equilibrium state, there must exist at least two coalitions that can decide to merge to improve their overall coalition value. As long as such two coalitions exist, the coalition structure changes to another coalition structure or else this procedure stops at an equilibrium state.

\section{B. Simulation Results}

For simulation illustrations, the following distributed CR network is set up: $n$ CRs are uniformly and independently distributed in a circle with radius $R_{S}=1000 \mathrm{~m}$ and centered at the coordinates $(\beta, 0)$. The PU is at coordinates $(0,0)$ as shown in Fig.1. The sensing time $\tau_{s}=1 \mathrm{~ms}$, the timebandwidth product is set as $N=6000$, and the slot duration is set to be $T_{f}=100 \mathrm{~ms}$. For path loss, we set $\alpha=3$. The PU power $P_{i}$, scalar $\kappa$ and noise power $\sigma_{i}^{2}$ are set at a value such that $\gamma_{i}$ (PU's SNR at CR $i$ ) at the coordinates $(\beta, 0)=(2000,0)$ is $-15 \mathrm{~dB}$. The probability of PU present is assumed to be $P_{H_{1}}=0.2$. To keep our simulation analysis simple, we assume that all the CRs have the same transmission rate, i.e., $r_{i}=r=\log \left(1+S N R^{s}\right)=3.4594 \mathrm{bits} / \mathrm{sec} / \mathrm{Hz}$ in (12), where $S N R^{s}$ is signal-to-noise ratio from a CR to its receiver. Simulations were performed by "dropping" the CRs randomly around the coordinates $(\beta, 0)$.

For the target detection probability $\tilde{P}_{d}=0.99$, in Fig. 2, we show the average (averaged over the simulation runs) throughput per CR for different network sizes, when all CRs sense independently (non-cooperative strategy), and when CRs can form coalitions (selfish and altruistic). It can be seen from Fig. 2 that the proposed coalition formation (both selfish and altruistic) yields an improvement in the average throughput as compared to the non-cooperative solution. However, the selfish coalition formation solution leads to a loss in average throughput as compared to the altruistic coalition formation solution. In Fig. 3, we compare the performance of altruistic and selfish coalition formation solutions with the non-cooperative solution in terms of average false alarm probability per CR for different network sizes. It can be seen that the altruistic coalition formation solution significantly reduces the average false alarm per $\mathrm{CR}$, as compared to both selfish coalition formation and noncooperative solutions. It can also be seen from Fig. 2 and 3 that an SNR dependent target detection probability for individual CRs in a coalition results in better average throughput and reduced average false alarm per CR as compared to when each $\mathrm{CR}$ is assigned the same target detection probability. In Fig. 4, we compare the performance of altruistic and selfish coalition formation solutions in terms of average throughput per CR for different values of $\tau_{c}$. In this figure, we show that for different values of $\tau_{c}$ the altruistic coalition formation solution yields significant average throughput gains when compared to the selfish coalition formation solution. In Fig. 5 we show average maximum coalition sizes for different values of $\tau_{c}$. It can be seen that for large values of $\tau_{c}$, the network structure is mostly composed of small coalitions.

\section{Conclusions}

We apply a coalitional game theoretic framework to the study of stable network partitions for the sensing-throughput tradeoff problem in distributed cognitive radio networks. We show that a coalition formation game for the sensingthroughput tradeoff problem is a non-transferable utility game 


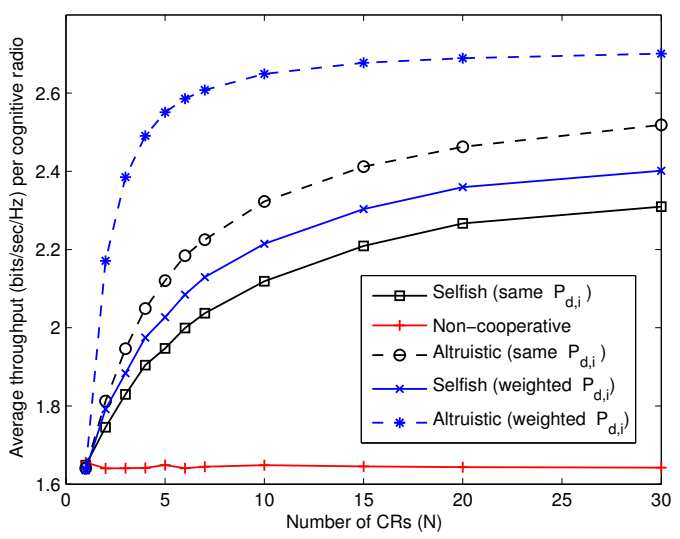

Fig. 2. Average throughput $[\mathrm{bits} / \mathrm{sec} / \mathrm{Hz}]$ per $\mathrm{CR}, \tilde{P}_{d}=0.99, \beta=2000 \mathrm{~m}$ and $\tau_{c}$ in (12) is set to $0.001 \mathrm{~ms}$. Given a target detection probability for a coalition, "same $P_{d, i}$ " means that each CR is required to satisfy the same target probability of detection (see equation 7), whereas "weighted $P_{d, i}$ " means that each CR is required to satisfy an SNR-dependent target detection probability (see equation 8).

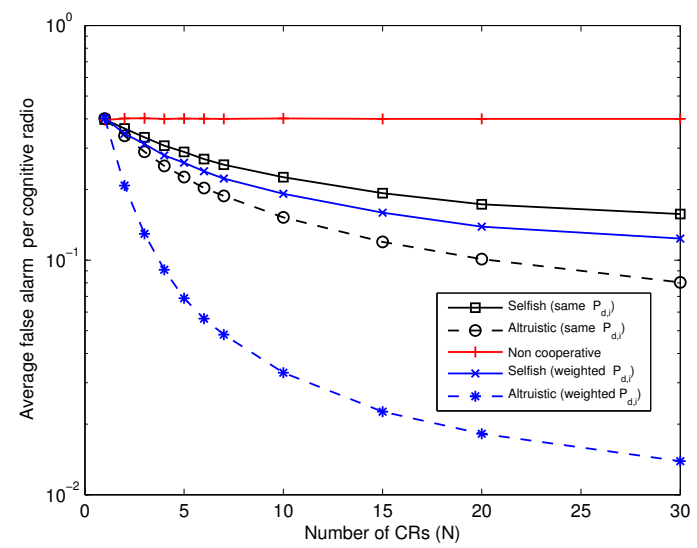

Fig. 3. Average false alarm probability per CR for different network sizes, $\tilde{P}_{d}=0.99, \tau_{c}$ (the time spent for reporting a sensing decision to the coalition head) in (12) is set to $0.001 \mathrm{~ms}$ and $\beta=2000 \mathrm{~m}$.

where each cognitive radio will have its own value within a coalition. We demonstrate that the altruistic coalition formation solution yields significant gains in terms of reduced average false alarm probability and increased average throughput per $\mathrm{CR}$ as compared to the selfish and non-cooperative solutions. Our work also analyzes the equilibrium state (where no two coalitions have an incentive to merge anymore) of the proposed coalition formation solution. We also show that an SNR-dependent target detection probability for individual CRs in a coalition results in better average throughput per CR as compared to when each CR is assigned the same target detection probability in a coalition.

\section{REFERENCES}

[1] Y.-C . Liang, Y. Zeng, E. C. Y. Peh, and A. T. Hoang, "Sensingthroughput tradeoff for cognitive radio networks." IEEE Transactions on Wireless Communications, vol. 7, no. 4, pp. 1326-1337, 2008.

[2] S. M. Mishra, A. Sahai, and R. W. Brodersen, "Cooperative sensing among cognitive radios," in Proc. IEEE ICC, 2006, pp. 1658-1663.

[3] R. B. Myerson, Game Theory: Analysis of Conflict. Philadelphia, PA, USA: Harvard University Press, 1997.

[4] D. C. Arney and E. Peterson, "Cooperation in social networks: Communication, trust, and selflessness." in Proc. 2008 Army Science Conference, 2008

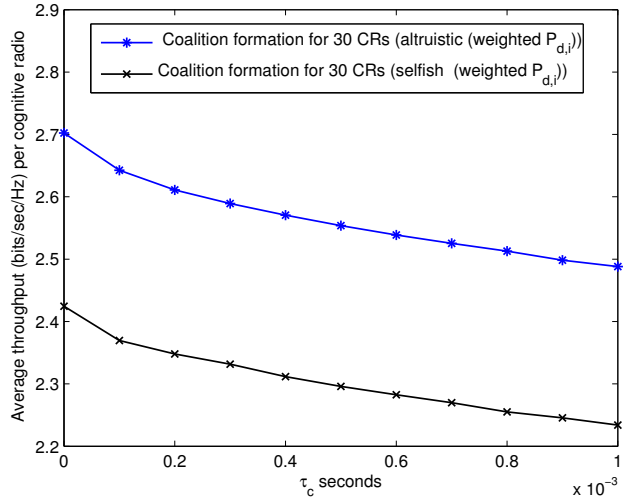

Fig. 4. Average throughput [bits/sec/Hz] per $\mathrm{CR}$ for selfish and altruistic coalition formation solutions, when the value of $\tau_{c}$ in (12) is varied between $0 \mathrm{~ms}$ to $1 \mathrm{~ms}, \tilde{P}_{d}=0.99$ and $\beta=2000 \mathrm{~m}$.

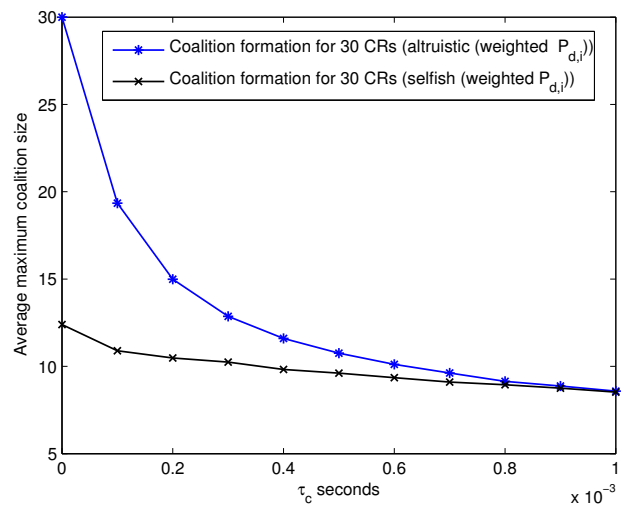

Fig. 5. Average maximum coalition size for selfish and altruistic coalition formation solutions, when the value of $\tau_{c}$ in (12) is varied between $0 \mathrm{~ms}$ to $1 \mathrm{~ms}, \tilde{P}_{d}=0.99$ and $\beta=2000 \mathrm{~m}$.

[5] W. Saad, Z. Han, M. Debbah, A. Hjørungnes, and T. Basar, "Coalitional games for distributed collaborative spectrum sensing in cognitive radio networks," in Proc. IEEE INFOCOM, Rio de Janeiro, Brazil, 2009.

[6] B. Wang, K. Liu, and T. Clancy, "Evolutionary game framework for behavior dynamics in cooperative spectrum sensing," in Proc. IEEE GLOBECOM 2008., 30 2008-Dec. 4 2008, pp. 1-5.

[7] S. Cordeiro, E. Sofer, and G. Chouinard, "Functional requirements for the 802.22 wran standard r47," Jan 2006.

[8] Q. Zhao, L. Tong, A. Swami, and Y. Chen, "Decentralized cognitive mac for opportunistic spectrum access in ad hoc networks: A pomdp framework," IEEE Journal on Selected Areas in Communications, vol. 25 , no. 3, pp. 589-600, 2007.

[9] R. Fan and H. Jiang, "Optimal multi-channel cooperative sensing in cognitive radio networks," to appear in IEEE Transactions on Wireless Communications, 2010.

[10] E. Peh and Y.-C. Liang, "Optimization for cooperative sensing in cognitive radio networks," in Proc. IEEE Int. Wireless Commun. Networking Conf., Hong Kong, Mar 2007, pp. 27-32.

[11] A. Ghasemi and E. S. Sousa, "Spectrum sensing in cognitive radio networks: the cooperation-processing tradeoff," Wireless Communications and Mobile Computing, vol. 7, no. 9, pp. 1049-1060, 2007.

[12] Z. Chair and P. K. Varshney, "Optimal data fusion in multiple sensor detection systems," IEEE Trans. Aerospace Electronic Syst, vol. 22, no. 1 , pp. 98-101, Nov. 1986.

[13] J. Unnikrishnan and V. Veeravalli, "Algorithms for dynamic spectrum access with learning for cognitive radio," IEEE Transactions on Signal Processing, vol. 58, no. 2, pp. 750-760, Feb. 2010

[14] S. Zhang, T. Wu, and V. N. Lau, "A low-overhead energy detection based cooperative sensing protocol for cognitive radio systems," IEEE Transactions on Wireless Communications, vol. 8, no. 11, pp. 55755581, Nov. 2009

[15] Z. Khan, S. Glisic, L. DaSilva, and J. Lehtomaki, "Modeling the dynamics of coalition formation games for cooperative spectrum sharing in an interference channel," under review, 2010. 